\title{
DIAGNOSTICS
}

\section{Validation of a simplified grading of Gram stained vaginal smears for use in genitourinary medicine clinics}

\author{
C A Ison, P E Hay
}

Sex Transm Infect 2002;78:413-415

See end of article for authors' affiliations

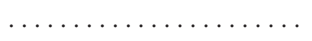

Correspondence to: Dr C A Ison, Medical Microbiology, Faculty of Medicine, Imperial College, St Mary's Campus, Norfolk Place, London W2 1PG, UK; c.ison@ic.ac.uk

Accepted for publication 5 July 2002

\begin{abstract}
Objectives: To validate a simplified grading scheme for Gram stained smears of vaginal fluid for the diagnosis of bacterial vaginosis (BV) against the accepted "gold" standard of Amsel's composite criteria.

Methods: Women attending genitourinary medicine (GUM) clinics, as part of a multicentre study, were diagnosed as having BV if three or more of the following criteria were present; homogeneous discharge, elevated vaginal $\mathrm{pH}$, production of amines, and presence of "clue" cells. Women with less than three of the criteria were considered as normal. Simultaneously, smears were made of vaginal fluid and Gram stained and then assessed qualitatively as normal (grade I), intermediate (grade II), or consistent with BV (grade III). Two new grades were used, grade 0, epithelial cells only with no bacteria, and grade IV, Gram positive cocci only.

Results: BV was diagnosed in 83/162 patient visits using the composite criteria, the remainder being regarded as normal. The majority of patients with BV had a smear assessed as grade III $(80 / 83,96 \%)$ and the majority of normal women had a smear assessed as grade I (normal, 48/79, 61\%), giving a high sensitivity $(97.5 \%)$, specificity $(96 \%)$, and predictive value for a positive $(94.1 \%)$ and negative $(96 \%)$ test, kappa index $=0.91$. Smears assessed as grade II were found predominantly (12/13) among patients diagnosed as normal, with less than three of the composite criteria. Grades 0 and IV were both only found among normal women.

Conclusion: This simplified assessment of Gram stained smears can be used as an alternative to Amsel's criteria and is more applicable for use in busy GUM clinics.
\end{abstract}

B acterial vaginosis $(\mathrm{BV})$ is a clinical entity that is characterised by a change in vaginal ecology where the normal flora of lactobacillus morphotypes is replaced by a mixed microbial flora consisting of anaerobes and Gardnerella vaginalis. ${ }^{1}$ The original description in 1955 by Gardner and Dukes $^{2}$ remains an accurate description of the presenting clinical features of a malodorous, thin homogeneous vaginal discharge. However, a variety of methods were used for diagnosis until 1984 when a consensus was reached to define the diagnosis of BV using the composite criteria described by Amsel et al. ${ }^{3}$ These included a thin homogeneous discharge, elevated vaginal $\mathrm{pH}$ above 4.5 , release of amines on the addition of $10 \%$ potassium hydroxide to vaginal fluid, and the presence of "clue" cells, of which three need to be present for the diagnosis of BV.

An alternative method of diagnosis that has been used extensively, particularly in research studies, is the grading (or scoring) of the microbial flora in Gram stained smears of vaginal fluid. This method reflects both the change in vaginal ecology and the strong microbial associations, and was first described by Spiegel et $a l^{4}$ in 1983. This initial report divided smears into those with normal lactobacillus morphotype flora and those with mixed flora consistent with BV. The method was modified by Nugent et $a l^{5}$ to include an intermediate category that demonstrated the presence of a mixed microbial flora but with significant numbers of the lactobacillus morphotype. Both of these methods score the smears by quantification of the different morphotypes that requires considerable time and skill and simpler versions have been described where the categories are assessed qualitatively ${ }^{67}$ In genitourinary medicine (GUM) clinics, at least in the United Kingdom, microscopy is in routine use as a method of diagnosis of STIs but there is neither the time nor sufficient expertise available to use the quantitative scoring systems. The aim of this study was to validate one of the simpler grading schemes for Gram stained smears previously described by Hay et $a l^{7} 8$ against the composite criteria ${ }^{3}$ for use in GUM clinics.

\section{METHODS}

Patients and collection of specimens

Patients included in this study were part of an investigator blind, randomised, parallel group, multicentre, phase IV treatment study comparing metronidazole gel and clindamycin cream. All patients entered into the study were clinically diagnosed as having BV using Amsel's criteria. Patients were recruited during the first 7 days after menstruation (visit 1) and were asked to return to the clinic between 12-16 days after commencing treatment (visit 2) and on the corresponding day of their next menstrual cycle, typically 26-35 days after starting drug treatment, (visit 3). At each visit, during the vaginal examination, a smear of vaginal fluid collected from the lateral fornix was prepared by the investigating clinician and sent to a single centre (Imperial College) for Gram staining and reading. A total of 162 smears were examined from 72 women, of which 16 attended for a single visit, 22 for two visits, and 34 for three visits.

\section{Grading of slides}

Unstained smears were received from each centre by post and were allocated a number, blinding the patient number, clinic visit, and referring centre to the reader. Smears were then Gram stained using the following protocol; smears were flooded with crystal violet (Pro-lab Diagnostics) for 30 seconds, washed in tap water, flooded with Lugol's iodine (Pro-lab Diagnostics) for 30 seconds, washed in water and then decolourised in acetone for 5-10 seconds, and finally counterstained with 15\% carbol fuchsin (Pro-lab Diagnostics) in neutral red (Pro-lab Diagnostics) for 1 minute. Smears were blotted dry and examined under oil immersion at ×1000 magnification. 
Table 1 Grading of Gram stained vaginal smears in women with and without bacterial vaginosis as defined by Amsel's composite criteria

\begin{tabular}{cccc}
\hline \multicolumn{4}{c}{ Amsel's criteria } \\
\cline { 2 - 4 } & Normal & BV & Total \\
\hline Grading of Gram stained smear & & \\
O & 6 & 0 & 6 \\
I & 48 & 2 & 50 \\
II & 12 & 1 & 13 \\
III & 5 & 80 & 85 \\
IV & 8 & 0 & 8 \\
Total & 79 & 83 & 162 \\
\hline
\end{tabular}

Smears were graded in the following manner as described previously $^{78}$ : grade I (normal flora), lactobacillus morphotype only; grade II (intermediate flora), reduced lactobacillus morphotype with mixed bacterial morphotypes; grade III (BV), mixed bacterial morphotypes with few or absent lactobacillus morphotypes. Two additional grades were also used; grade 0 , epithelial cells with no bacteria seen and grade IV, epithelial cells covered with Gram positive cocci only.' All slides were read by a single reader and no systematic quality control was in place. However, after the initial analysis, all discrepant slides were reread (blinded to the original result) and gave identical results.

\section{Analysis of data}

Sensitivity, specificity, and predictive values for a positive and negative test were used to compare grading of Gram stained smears against the composite criteria for the diagnosis of BV. The measure of agreement was determined by kappa index where a value of 1.0 indicates complete agreement. Smears scored as grade I (normal) were considered negative, grade III (consistent with BV) were considered positive, and grade II (intermediate) were analysed as both negative and positive.

\section{RESULTS}

Women attending for 162 patient visits were divided into those without BV (79), who were considered normal, and those with BV (83) by the composite criteria. Gram stained smears of vaginal fluid collected at each of these patient visits were graded into one of five grades. Women with and without BV were subdivided by the grading of their Gram stained smear (table 1). The majority of patients without BV had smears assessed as grade I $(48 / 79,61 \%)$ and patients with BV had smears assessed as grade III $(80 / 83,96 \%)$. The intermediate flora (grade III) was found among 12/79 (15\%) women without BV and only 1/83 (1\%) women with BV. Smears graded as $0(6 / 79,8 \%)$ or IV $(8 / 79,10 \%)$ were only found among normal women.

The sensitivity of grading Gram stained smears for the diagnosis of $\mathrm{BV}$ was found to be $\geqslant 96 \%$ when grade II was considered either as negative or positive or was excluded (table 2). Similarly, the predictive value of a negative test was high in each analysis. The specificity and the predictive value of a positive test were also found to be high, $90.5 \%$ and $94.1 \%$ respectively, when grade II was considered negative or was excluded from the analysis but were reduced if grade II was considered positive to $73.8 \%$ and $82.6 \%$ respectively (table 2 ).

$\mathrm{BV}$ as diagnosed by the composite criteria versus this simplified grading scheme gave a kappa value of 0.91 if smears graded as intermediate (grade II) were considered negative, whereas a value of 0.76 was obtained if grade II smears were considered positive. Of the 13 patients whose smears were graded as intermediate, six patients had none, four patients had one, two had two, and one had four of the composite criteria.
Table 2 Sensitivity, specificity, and predictive values for grading of Gram stained smears for the diagnosis of bacterial vaginosis using Amsel's criteria as the gold standard

\begin{tabular}{llll}
\hline & 1 & 2 & 3 \\
\hline Sensitivity & 97.5 & 96.0 & 97.5 \\
Specificity & 90.5 & 90.5 & 73.8 \\
Predictive value for positive & 94.1 & 94.1 & 82.6 \\
Predictive value for a negative & 96.0 & 96.0 & 96.0
\end{tabular}

1 = Smears: grade I = negative; grade $\mid \mathrm{II}=$ positive; grade $\mathrm{I}$ = excluded.

2 = Smears: grades I, $\|$ = negative; grade $I I I=$ positive

3 = Smears: grade $\mid=$ negative; grades $I I, I I=$ positive

NB: 6 smears graded as 0 (all had $<3$ Amsel's criteria); 8 smears graded as IV (all had <3 Amsel's criteria)

\section{DISCUSSION}

The aim of this study was to validate a simplified grading of Gram stained vaginal smears for the diagnosis of BV in GUM clinics. The composite criteria described by Amsel et al were used as the reference method as this is still regarded as the "gold" standard. The patients were a highly selected group and no prevalence data were obtained but the patient visits divided almost equally into normal and BV giving a good basis for this analysis. There was a strong association of grade I flora with normal women and of grade III with BV with resulting high sensitivity, specificity, and predictive values which were similar or higher than other grading schemes compared to the composite criteria. $^{6}{ }^{10}$ In an international comparison of different scoring methods our simplified method also equated well with the scoring described by Nugent et al (kappa: 0.89) which is considered the reference method for reading Gram stained smears. ${ }^{11}$ These results indicate that when there is a lack of time or expertise, this qualitative assessment of the microbial flora can be used as an alternative method of diagnosis.

Smears with grade II or intermediate flora were found most commonly in normal women in this study. Intermediate flora have been shown to consist of bacteria associated with BV, such as $G$ vaginalis and anaerobes, but in addition to have significant numbers of lactobacilli usually associated with normal flora and are believed to be a transient phase between normal and $\mathrm{BV}{ }^{12}{ }^{13}$ This study suggests that, although this may be true, it does not produce the full clinical criteria of BV.

Smears that were graded as 0 had no bacteria present suggesting the presence of an antibacterial agent in the vagina. While this will only be seen occasionally, it may become more common as vaginal creams and gels are used increasingly. Smears that were graded as IV demonstrated only Gram positive cocci and were found in normal women. There is no evidence that these flora are associated with BV or are abnormal.

\section{ACKNOWLEDGEMENTS}

We would like to thank the following consultants and their staff for recruiting patients and collection of smears, Stephen Tchamouroff, Brighton; Christine Bowman, Nottingham; Joyti Dhar, Derby; Vincent Riley, Leicester; Angela Robinson, Mortimer Market, London; Jean Tobin, Portsmouth; Fred Wilmott, Southampton; Andrew Nayagam, Shoreham on Sea, West Sussex; S Uthayakumar, Stevenage. This work was funded by $3 \mathrm{M}$ Health Care Ltd.

Conflicts of interest: none.

\section{CONTRIBUTORS}

CAI contributed to the study design and was responsible for reading the smears, analysis of the data, and preparation of the manuscript; $\mathrm{PEH}$ contributed to study design, recruiting patients, and analysis of the data. 


\section{Authors' affiliations}

C A Ison, Department of Infectious Diseases and Microbiology, Faculty of Medicine, Imperial College, St Mary's Campus, London, UK P E Hay, Department of Genitourinary Medicine, St George's Hospital Medical School, London, UK

\section{REFERENCES}

1 Pheifer TA, Forsyth PS, Durfee MA, et al. Nonspecific vaginitis: role of Haemophilus vaginalis and treat ment with metronidazole. N Engl J Med 1978:298: 1429-34

2 Gardner HL, Dukes $C D$. Haemophilus vaginalis vaginitis. A newly defined specific infection previously classified 'nonspecific' vaginitis. Am J Obstet Gynecol 1955;69:962-76.

3 Amsel R, Totten PA, Spiegel CA, et al. Nonspecific vaginits. Diagnostic criteria and microbial and epidemiologic association. Am J Med 1983;74:14-22.

4 Spiegel CA, Amsel R, Holmes KK. Diagnosis of bacterial vaginosis by direct Gram stain of vaginal fluid. J Clin Microbiol 1983;18:170-7.

5 Nugent RP, Krohn MA, Hillier SL. Reliability of diagnosing bacteria vaginosis is improved by a standardized method of Gram stain interpretation. J Clin Microbiol 1991;29:297-301.

6 Thomason JL, Anderson R, Gelbart SM, et al. Simplified Gram stain interpretative method for diagnosis of bacterial vaginosis. Am J Obste Gynecol 1992;167:16-19.
7 Hay PE, Lamont RF, Taylor-Robinson D, et al. Abnormal bacterial colonisation of the lower genital tract and subsequent preterm delivery and late miscarriage. BM 1994;308:295-8.

8 Hay PE, Morgan DJ, Ison CA, et al. A longitudinal study of bacterial vaginosis during pregnancy. Br J Obstet Gynaecol 1994;101 1048-53.

9 Bacterial Special Interest Group of the MSSVD. Vaginal flora. In: Ison CA, Savage M, Taylor-Robinson D, eds. Microscopy of sexually transmitted infections. London: Harcourt Health Communications, 2001: Chapter 5:19.

10 Schwebke JR, Hillier SL, Sobel JD, et al. Validity of the vaginal Gram stain for the diagnosis of bacterial vaginosis. Obstet Gynecol 1996:88:573-6.

11 Forsum U, Larsson PG, Schmidt $\mathrm{H}$, et al. The deviance (inter-observer variation) between the interpretations of Gram staining criteria of BV made by the participants of the BVOO workshop. BV 2000, International meeting on Bacterial vaginosis. 2000 (abstract).

12 Hillier SL, Krohn MA, Nugent RP, et al, for the vaginal infections and prematurity study group. Characteristics of three vaginal flora patterns assessed by Gram stain among pregnant women. Am J Obstet Gynecol 1992; 166:938-44

13 Rosenstein IJ, Morgan DJ, Sheehan M, et al. Bacterial vaginosis in pregnancy: distribution of bacterial species in different gram-stain categories of the vaginal flora. J Med Microbiol 1996;45:120-6.

\section{NEW STI ONLINE SUBMISSION AND REVIEW SYSTEM}

I am pleased to inform authors and reviewers that as of 21 February 2002, STI will be using a new online submission and review system. Developed by Highwire Press (CA, USA), Bench>Press is a fully integrated electronic system which utilises the web to allow rapid and efficient submission of manuscripts. It also allows the peer review process to be conducted entirely online. The aim, apart from saving trees, is to speed up the frequently frustrating progress from submission to publication.

Authors can submit their manuscript in any standard word processing software. Standard graphic formats acceptable are: .jpg, .tiff, .gif, and eps. (nb. multipage powerpoint files are NOT acceptable). The text and graphic files are automatically converted to PDF for ease of distribution and reviewing purposes. Authors are asked to approve their submission before it formally enters the reviewing process. On approval by the authors, the submission is passed to the editor and/or reviewers via the web. All transactions are secure.

To access the system click on "SUBMIT YOUR MANUSCRIPT HERE" on the STI homepage: http://www.sextransinf.com/ or you can access Bench>Press directly at http://submit-sti.bmijournals.com/.

We are very excited with this new development and I would encourage authors and reviewers to use the online system where possible. It really is simple to use and should be a big improvement on the current peer review process. Full instructions can be found on Bench>Press and STI online. Please contact Natalie Davies, Project Manager, ndavies@bmigroup.com for further information.

\section{PRE-REGISTER WITH THE SYSTEM}

We would be grateful if all Sexually Transmitted Infections authors and reviewers pre-registered with the system. This will give you the opportunity to update your contact and expertise data, allowing us to provide you with a more efficient service.

\section{Instructions for registering}

1. Enter http://submit-sti.bmijournals.com

2. Click on "Create a New Account" in the upper left hand side of the Bench>Press homepage

3. Enter your email address in the space provided.

4. Choose a password for yourself and enter it in the spaces provided.

5. Complete the question of your choice to be used in the event you cannot remember your password at a later time.

6. Click on the "Save" button at the bottom of the screen.

7. Check the email account you registered under. An email will be sent to you with a verification number and URL.

8. Once you receive this verification number, click on the URL hyperlink and enter the verification number in the relevant field. This is for security reasons and to check that your account is not being used fraudulently.

9. Enter/amend your contact information, and update your expertise data. 